\title{
The Higgs bosons decays in the NMSSM with CP-violation
}

\author{
A.V. Gurskaya ${ }^{1, \star}$ and M.V. Dolgopolov ${ }^{1, \star \star}$ \\ ${ }^{1}$ Samara University, Academician Pavlov 1, 443011 Samara, Russia.
}

\begin{abstract}
We investigate the Higgs bosons decay width in the framework of NMSSM in the one-loop approximation. The Higgs sector under consideration contains CP-violation in the general case due to explicit and spontaneous violations of the CP-symmetry. The local minimum conditions for the Higgs bosons physical states are taken into account as necessary requirements. So, calculation of the decay width reduces to the calculation of corresponding processes amplitudes. The strategy of calculation is based on the perturbation theory.
\end{abstract}

\section{Introduction}

The Higgs sector is the main object of our research. The Higgs boson with mass $125 \mathrm{GeV}$ is observed in the experiments at the LHC. There are no signals of other particles that could be Higgs bosons with smaller masses. Why do we extend the Higgs sector?

First, the Higgs sector is responsible for the masses of fundamental particles. But there is a discrepancy between the SM predictions and neutrino oscillation experiments. We know that different types of neutrino transit into each other $[1,2]$. Therefore, the neutrino has mass. Since the SM does not explain this problem, then the Higgs sector of this model is still under consideration in this sense.

Secondly, there is the vacuum stability problem. There are researches [3, 4], in which it is considered that the vacuum is the metastable state for the Higgs boson with mass of order $125 \mathrm{GeV}$. Then, we need to understand where the "island of stability" is and for what values of physical parameters it exists?

The third problem is the baryon asymmetry justification. There is a hypothesis that the baryon asymmetry appear already at an early stage of the Universe formation in the framework of process, so-called, baryogenesis. It is the formation of hadrons from quarks and gluons. One of the condition of baryon asymmetry is combined violation of $\mathrm{C}$ - and $\mathrm{P}$ - parity (CP-violation) [5]. The SM contains the complex CP-violating-phase in the fundamental fermions mix matrix. However, this phase is not enough to explain of the baryon asymmetry. May be, there are additional CP-violation sources in the Higgs sector. It is reason for we need to extend the Higgs sector [6, 7].

Note also that the supersymmetric models contain extended Higgs sector naturally. There are requirements of supermultiplets chirality. It is leading to the replacing the hermitian conjugate doublet in the new doublet. We can not realize supersymmetry in SM because the degrees of freedom for bosons and fermions states are not the same. The simplest susy-model is MSSM, but it has so-called

\footnotetext{
${ }^{\star}$ e-mail: a-gurska@yandex.ru

$\star \star$ e-mail: mvdolg@yandex.ru
} 
$\mu$-problem. The solution of this problem is the introduction of the additional Superpole chiral singlet in the Higgs sector. This model is called Next-to-MSSM or NMSSM [8, 9].

\section{Higgs bosons in NMSSM with CP-violation}

In order to introduce the CP-violation in our model, we need to rewrite the complex structure in Lagrangian (complex couplings or complex vacuum expectation values parameters). Note also, the supersymmetry is broken, otherwise superparticles would have the same masses as their partners in the SM. Terms, which violate the supersymmetry, also violate CP-symmetry.

The CP-violation case in the NMSSM was considered by author in earlier work [10]. There is also a similar work dedicated to the potential of the Higgs NMSSM with explicit CP-violation [11]. In general case the explicit $\mathrm{CP}$-violation and spontaneous violation of $\mathrm{CP}$-symmetry take place. In order to calculate physical states of the Higgs bosons, we have to find the local minimum conditions of Higgs potential. This procedure is necessary for finding stable vacuum states. So, for it, we found the first and second derivations of potential on the fields in the minimum. We need to diagonalize of the $5 \times 5$ mass squared matrix of neutral Higgs bosons, and does not have viable analytical solutions. The eigenstates considered to be positive in local minimum.

The local minimum requirements fix all parameters ranges and set fixed parameters like valid scenarios. Several scenarios compare with CP-conserving case were considered in [10]. the scenario with the first Higgs boson mass less then $125 \mathrm{GeV}$ is of the principal interest. We are interested in this scenario in the context of the baryon asymmetry problem and baryogenesis. There is the hypothesis of electroweak baryogenesis, in which a strong phase transition of the first order is required. And this phase transition is realized for the Higgs bosom with the mass less than $50 \mathrm{GeV}$. The considered scenario satisfies this hypothesis.

\section{Higgs bosons decay width calculation}

We will consider $1 \rightarrow 2$ process. General formula for this case is well known:

$$
\Gamma(H \rightarrow X \bar{X})=\frac{\left|A_{H \rightarrow X \bar{X}}(\varphi)\right|^{2}}{16 \pi M_{H}} \sqrt{1-\frac{4 m_{X}^{2}}{M_{H}^{2}}},
$$

where $M_{H}$ - Higgs boson mass, $m_{X}$ - final particle mass, $A_{H \rightarrow X \bar{X}}(\varphi)$ - the process amplitude, depending on CP-violation phase.

And we consider decay into vector bosons and into fermions (see Fig.1).

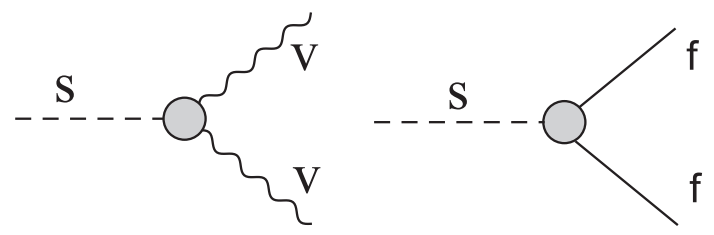

Fig. 1. Two types for decay of scalar particle without definition of loop topology.

Choosing the method of calculating the next perturbation order, we can represent the results of calculations close to the full set of loop contributions. The advantage of this approach is that the computation reduces to the minimum set of scalar integrals, and those, in turn, are expressed through 
known functions of the Veltman-Passarino A0, B0, C0 [12, 13]. The calculation of the vertex functions in the appropriate order of perturbation theory in the framework of this approach should be limited only to the numerical calculation of the reduced analytical results for the select set of generalized structures that contributed to this vertex function, bypassing the stage tensor, dimensional and algebraic reduction $[13,14]$.

Diagrams on Fig.1 present general case for decay of scalar particle. In particular, we have five types of diagrams for final states (see Fig. 2, 3).

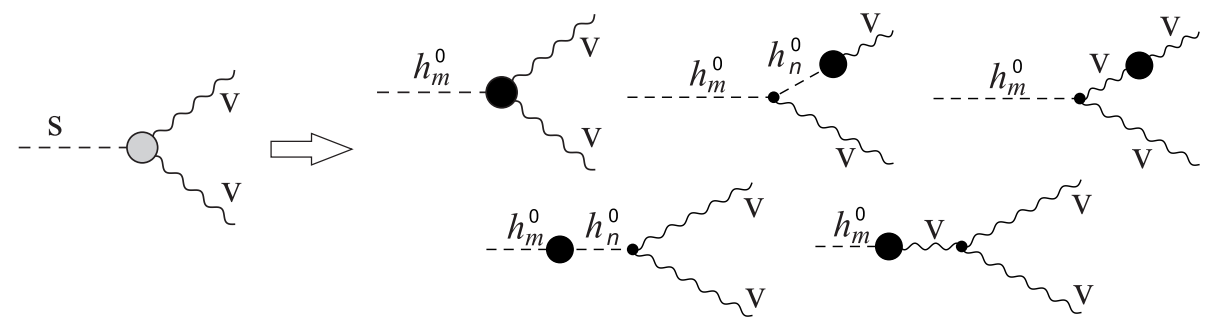

Fig.2. Diagrams of the neutral Higgs bosons decay to the gauge.

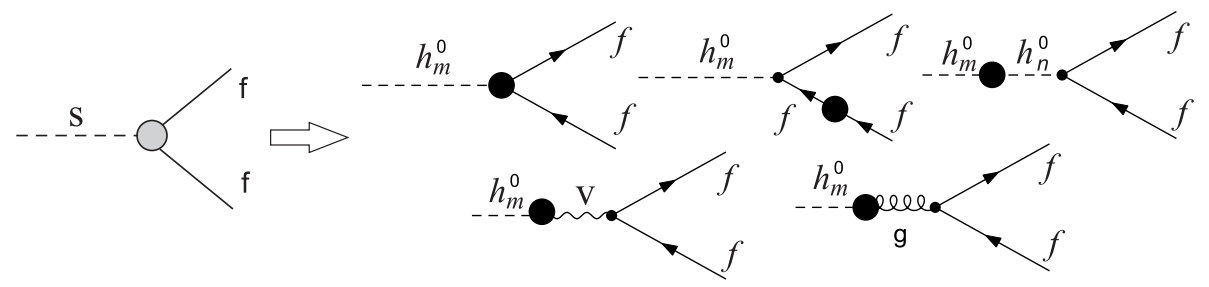

Fig 3. Diagrams of the neutral Higgs bosons decay to the fermions.

For example, there is the diagram type, when the Higgs boson has decay into any vector particles. We may define it like this (Fig 4):

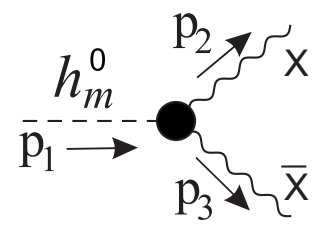

Fig.4. Diagram of the scalar particle decay into the vector particles.

Formula for this one-loop correction is given by

$$
\left.\begin{array}{rl}
T^{s v v}=\epsilon^{\mu v \sigma \rho} & p_{2 \sigma} p_{3 \rho} G_{h_{m} X \bar{X}}^{(1)}+g^{\mu v} G_{h_{m} X \bar{X}}^{(2)}+p_{2}^{\mu} p_{2}^{v} G_{h_{m} X \bar{X}}^{(3)} \\
& +p_{3}^{\mu} p_{3}^{v} G_{h_{m} X \bar{X}}^{(4)}+p_{2}^{\mu} p_{3}^{v} G_{h_{m} X \bar{X}}^{(5)}+p_{3}^{\mu} p_{2}^{v} G_{h_{m} X \bar{X}}^{(6)}
\end{array}\right\}
$$

Each factor has analytical expression. For example, first $G^{(1)}$ : 


$$
G_{h_{m} X \bar{X}}^{(1)}=-\frac{i N_{c} K}{4 \pi^{2}}\left[F^{(1)} m_{1} C_{0}+\left(F^{(1)} m_{1}-F^{(2)} m_{2}\right) C_{1}+\left(F^{(1)} m_{1}+F^{(3)} m_{3}\right) C_{2}\right],
$$

where $F^{(1)}, F^{(2)}, F^{(3)}$ are defined from $s+p \gamma^{5}$ and $v \gamma^{\mu}+a \gamma^{\mu} \gamma^{5}$ as its combinations.

In the CP-violating case in the Higgs sector the scalar $(s)$ and pseudoscalar $(p)$ Yukawa couplings are following:

$$
\begin{gathered}
\bar{u} u h_{j}:-\frac{g M_{u}\left(\mathrm{~A}_{1 j} s_{\beta}-i \gamma^{5} \mathrm{~A}_{2 j} c_{\beta}+\mathrm{A}_{3 j} c_{\beta}\right)}{2 m_{W} s_{\beta}} \\
\bar{d} d h_{j}: \frac{g M_{d}\left(-\mathrm{A}_{1 j} c_{\beta}+s_{\beta}\left(\mathrm{A}_{3 j}+i \gamma^{5} \mathrm{~A}_{2 j}\right)\right)}{2 c_{\beta} m_{W}},
\end{gathered}
$$

where $A_{i j}$ - squared mass matrix components of CP-add and CP-even mixing between physical states.

We take into account the mixing between CP-states in two stages. The parametrization is given by:

$$
\begin{gathered}
H_{1}=\left(\begin{array}{c}
\frac{1}{\sqrt{2}}\left(v_{1}+\phi_{1}^{0}+i \chi_{1}\right) \\
\phi_{1}^{-}
\end{array}\right), H_{2}=e^{i \theta}\left(\begin{array}{c}
\phi_{2}^{+} \\
\frac{1}{\sqrt{2}}\left(v_{2}+\phi_{2}^{0}+i \chi_{2}\right)
\end{array}\right) \\
S=\frac{1}{\sqrt{2}} e^{i \varphi}\left(v_{3}+\phi_{3}^{0}+i \chi_{3}\right),
\end{gathered}
$$

where $v_{i}$ - the vacuum expectation of Higgs field; $\theta, \varphi$-spontaneous CP-violation phases.

First, we get the basis of fields with defined CP-parity $\left(H, h, A^{0}, G^{0}\right)$ :

$$
\left(\begin{array}{l}
\phi_{1}^{0} \\
\phi_{2}^{0}
\end{array}\right)=\left(\begin{array}{cc}
\cos \beta & -\sin \beta \\
\sin \beta & \cos \beta
\end{array}\right)\left(\begin{array}{c}
H \\
h
\end{array}\right) ;\left(\begin{array}{l}
\chi_{1} \\
\chi_{2}
\end{array}\right)=\left(\begin{array}{cc}
\cos \beta & -\sin \beta \\
\sin \beta & \cos \beta
\end{array}\right)\left(\begin{array}{c}
G^{0} \\
A^{0}
\end{array}\right)
$$

And then the second twist is following:

$$
\left(\begin{array}{c}
H \\
A^{0} \\
h \\
\phi_{3}^{0} \\
\chi_{3}
\end{array}\right)=A_{i j}\left(\begin{array}{l}
h_{1} \\
h_{2} \\
h_{3} \\
h_{4} \\
h_{5}
\end{array}\right),
$$

where $h_{i}$ - physical states without defined CP-parity.

\section{Summary and Outlook}

In the presented note we deal with the one-loop corrections to Higgs bosons decay widths in the framework of NMSSM. We considered combined CP-violation due to explicit and spontaneous violations in the Higgs sector. We conclude that the local minimum conditions of potential have the crucial role for the Higgs bosons physical states existence in agreement with allowed ranges of NMSSM parameters.

Authors are going to consider the temperature evolution of the Higgs potential in the next upcoming work. It will allow the range selection for free and control parameters of the model. Some analysis in the study of this problem was developed by methods of the catastrophes theory $[15,16]$. 


\section{References}

[1] Fukuda Y et al. (Super-Kamiokande Collab.) Phys. Rev. Lett. 811562 (1998); 822644 (1999); Fukuda S et al. (Super-Kamiokande Collab.) Phys. Rev. Lett. 853999 (2000)

[2] Hampel W et al. (GALLEX Collab.) Phys. Lett. B 447127 (1999); Altmann M et al. (GNO Collab.) Phys. Lett. B 49016 (2000); Bellotti E (for the GNO Collab.) Nucl. Phys. B: Proc. Suppl. 9144 (2001).

[3] Elias-Miro J. Higgs mass implications on the stability of the electroweak vacuum / J. Elias-Miro [et al.] // e-print: arXiv:1112.3022v1.

[4] Degrassi G. Higgs mass and vacuum stability in the Standard Model at NNLO / G. Degrassi [et al.] // e-print: arXiv:1205.6497v2.

[5] Sakharov A.D. Violation of CP invariance, C-asymmetry and baryon asymmetry of the Universe // Pisma v ZhETF, 1967, Vol. 5, Issue 1, 32-35.

[6] Dolgopolov M.V., Rykova E.N. Constraints on electroweak baryogenesis in models involving an extended Higgs sector // Physics of Atomic Nuclei Volume 72, Issue 1, Pages 173-177.

[7] Dolgopolov M.V., Dubinin M.N., Rykova E.N. Two-Higgs doublet potential of the MSSM at finite temperature and Higgs boson masses // Quarks. 2008. V. 1.

[8] Higgs bosons in a nonminimal supersymmetric model / J. Ellis [et al.] // Physics Review D. 1989. V.39. P.845-869.

[9] Maniatis, M. The Next-to-minimal supersymmetric extension of the standard model reviewed // IJMP A. 2010. V.25. P.3505-2602.

[10] Gurskaya A. Higgs bosons in NMSSM with CP-violation / Proceedings of the XXII International Workshop QFTHEP'2015 - MSU, SINP.

[11] T. V. Volkova, M. V. Dolgopolov, M. N. Dubinin, E. N. Rykova, Effective Higgs potential in Next-to-Minimal Supersymmetric Standard Model, Vestn. Samar. Gos. Tekhn. Univ. Ser. Fiz.Mat. Nauki, 2(31) (2013), 233242.

[12] 't Hooft G. Scalar one loop integrals / G. 't Hooft, M.Veltman // Nucl.Phys.B. 1979. V.153. 365-401.

[13] Passarino G. One loop correction for $e^{+} e^{-}$annihilation into $\mu^{+} \mu^{-}$in the weinberg model / G.Passarino, M.Veltman // Nucl.Phys.B. 1979. V.160. P.151.

[14] Philippov, Y.P. The new presentation of the algebraic reduction results for B0, C0 scalar integrals // Teoreticheskaya psizika. 2005. V.6. 86-97.

[15] M. N. Dubinin, E. Yu. Petrova, High-temperature Higgs potential of the two-doublet model in catastrophe theory, Teoret. Mat. Fiz., 184:2 (2015), 315-337.

[16] M. V. Dolgopolov, S. P. Zavodov, E. Yu. Petrova, Bifurcation sets of extended Higgs potential, Vestn. Samar. Gos. Tekhn. Univ. Ser. Fiz.-Mat. Nauki, 4(33) (2013), 173-183. 